\title{
Mixoma de átrio direito com origem na veia cava inferior: uma localização rara com implicações diagnósticas e terapêuticas
}

\author{
Noedir A. G. STOLF*, Anderson BENÍCIO*, Luiz Felipe P. MOREIRA*, Eduardo ROSSI*
}

RBCCV 44205-510

\begin{abstract}
Stolf N A G, Benício A, Moreira L F P, Rossi E - Mixoma de átrio direito com origem na veia cava inferior: uma localização rara com implicações diagnósticas e terapêuticas. Rev Bras Cir Cardiovasc 2000; 15(3):255-8.

RESUMO: Os mixomas são os tumores cardíacos primários mais freqüentes. Sua localização habitual é o átrio esquerdo, sendo encontrados também em outros locais. É relatado o caso de paciente de 71 anos que, com diagnóstico de tumor em átrio direito, foi submetido a operação para retirada do tumor. A operação foi realizada, sendo confirmado o diagnóstico e procedida a ressecção do tumor com sucesso e sem intercorrência. Em estudo ecocardiográfico de controle no 4으ês de pós-operatório, evidenciou-se presença de massa residual que parecia originar-se na veia cava inferior. Foi submetida a nova operação, em que foi realizada a ressecção do mixoma, que se originava na veia cava inferior e se projetava para o interior do átrio direito. Com o tumor, foi ressecada, na sua implantação, uma porção da veia cava inferior. O presente relato mostra uma localização rara de origem do mixoma, bem como as complicações quanto ao diagnóstico e à abordagem no tratamento cirúrgico.
\end{abstract}

DESCRITORES: Mixoma, cirurgia. Neoplasias cardíacas, cirurgia. Veia cava inferior. Mixoma, diagnóstico. Neoplasias cardíacas, diagnóstico. Veia cava inferior, cirurgia.

\section{INTRODUÇÃO}

Os mixomas são os tumores cardíacos primários benignos mais freqüentes. Aproximadamente $75 \%$ a $80 \%$ têm sua origem no átrio esquerdo; $10 \%$ a $20 \%$ no átrio direito e de $5 \%$ a $10 \%$ em ambos os átrios (1). Podem, no entanto, originar-se em outros locais, como aorta, artéria pulmonar, ventrículos, valvas cardíacas ou mesmo em outros órgãos, entre eles ossos e pulmões (2).
Os sinais e sintomas não são típicos ou específicos. Podem simular desde uma estenose e/ou insuficiência mitral até uma síndrome de Budd-Chiari (3). Essas variadas manifestações dependem do local e da extensão do tumor.

Métodos não invasivos, como a ecocardiografia e a tomografia computadorizada de tórax, trouxeram grande contribuição ao diagnóstico dos mixomas

Trabalho realizado no Instituto do Coração do Hospital das Clínicas da Faculdade de Medicina da Universidade de São Paulo. São Paulo, SP, Brasil. Recebido para publicação em dezembro de 1999.

*Do Instituto do Coração do Hospital das Clínicas da Faculdade de Medicina da Universidade de São Paulo.

Endereço para correspondência: Noedir A. G. Stolf. Av. Dr. Enéas de Carvalho Aguiar, 44, Divisão Cirúrgica. Cerqueira César. São Paulo, SP, Brasil. CEP: 05403-000. Tel: (11) 3069-5318.e-mail: stolf@incor.usp.br 
Stolf N A G, Benício A, Moreira L F P, Rossi E - Mixoma de átrio direito com origem na veia cava inferior: uma localização rara com implicações diagnósticas e terapêuticas. Rev Bras Cir Cardiovasc 2000; 15(3): 255-8.

intracardíacos. Além da confirmação do diagnóstico, permitem o estabelecimento da tática cirúrgica adequada.

O objetivo desta publicação é o relato do caso do paciente com mixoma que se origina da veia cava inferior, bem como ressaltar alguns aspectos importantes no diagnóstico e na abordagem cirúrgica dos mixomas intracardíacos, que se tornaram evidentes no caso.

\section{RELATO DO CASO}

Paciente de 71 anos, do sexo feminino, cor branca, apresentava quadro clínico de síncope e dispnéia aos médios esforços. Não se verificou alteração na radiografia de tórax ou no ECG. O estudo ecocardiográfico mostrou massa em átrio direito, que invadia a valva tricúspide e projetava-se para o interior do ventrículo direito. A tomografia computadorizada de tórax confirmava a presença da massa com as mesmas características. A paciente foi, então, submetida a operação. A circulação extracorpórea foi estabelecida pela canulação da aorta e das veias cavas inferior e superior. Foi estabelecida hipotermia de $28^{\circ} \mathrm{C}$ e a proteção miocárdica foi realizada pela infusão de solução cardioplégica sangüínea fria à $4^{\circ} \mathrm{C}$ com reperfusão quente.

Após a abertura do átrio direito observou-se massa tumoral de aspecto regular, lisa, móvel sem evidências de implantação na parede do átrio. $O$ exame do tumor não evidenciou, também, irregularidade que sugerisse base de implantação ou fragmentação. A valva tricúspide se encontrava em perfeito estado, sem nenhum sinal degenerativo ou de comprometimento pela massa. Não havia evidências de implantação do tumor nessa valva. Não houve intercorrência intra-operatória ou na evolução, até a alta hospitalar.
No acompanhamento ambulatorial, através de ecocardiografia de controle pós-operatório, no 4 은 mês de pós-operatório, evidenciou-se massa residual no átrio direito. Esse mesmo estudo repetido sugeriu que o tumor se originasse na veia cava inferior.

Realizou-se, então, nova operação e a tática cirúrgica empregada foi modificada. A linha arterial foi estabelecida pela canulação da aorta, porém a drenagem venosa foi estabelecida pela canulação da veia cava superior e da veia femoral direita. Foi realizada parada circulatória total em hipotermia profunda de $16^{\circ}$ C. Com a abertura do átrio direito e exposição ampla da veia cava inferior, observou-se que o tumor se originava na veia cava inferior junto ao óstio da veia supra-hepática (Figura 1A). Foi ressecada sua base de inserção junto à porção da veia cava, sem que houvesse fragmentação do tumor (Figuras 1B e 2). A reconstrução da veia cava foi realizada com a utilização de retalho de pericárdio bovino.

A paciente recebeu alta no 80 dia de pós-operatório, após evolução sem intercorrências ou complicações.

\section{COMENTÁRIOS}

Os mixomas representam $35 \%$ a $50 \%$ dos tumores cardíacos primários ${ }^{(4)}$. Apresentam grande diversidade de sintomas, os quais se relacionam com o tamanho e a posição que ocupam. Os sintomas mais freqüentes são: sintomas relacionados à falência ventricular direita, sintomas constitucionais (febre, perda de peso) e síncope. Outros também podem estar presentes: fraqueza, fadiga, dor torácica, palpitações, obstrução da veia cava superior, fenômenos embólicos sistêmicos e pulmonares. Podem também apresentar envolvimento das valvas mitral e tricúspide, simulando quadros de estenose e/ou insuficiência.
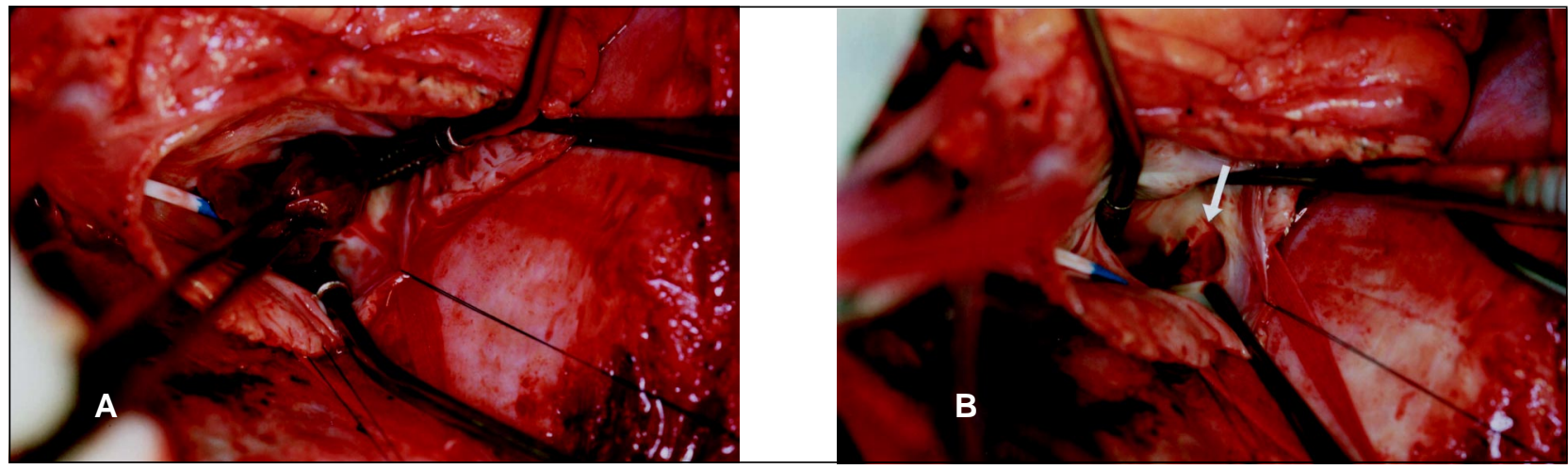

Fig. 1 - A- Tumor se originando na veia cava inferior junto ao óstio da veia supra-hepática. B- Massa tumoral com sua base de inserção. 
Stolf N A G, Benício A, Moreira L F P, Rossi E - Mixoma de átrio direito com origem na veia cava inferior: uma localização rara com implicações diagnósticas e terapêuticas. Rev Bras Cir Cardiovasc 2000; 15(3): 255-8.

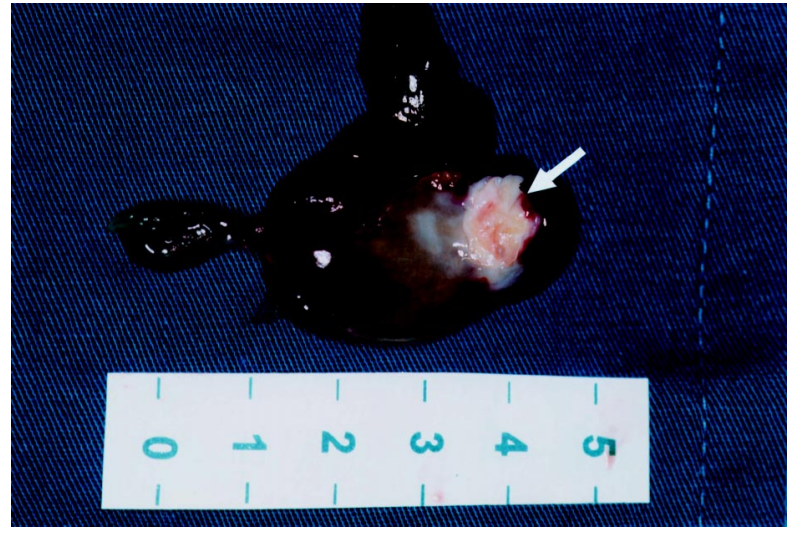

Fig. 2 - Base de inserção do tumor ressecada junto ao óstio da veia supra-hepática.

Os mixomas do átrio direito geralmente se originam na fossa oval ou na base do septo interatrial. A origem do mixoma na veia cava inferior é extremamente rara; encontramos na literatura o relato de apenas 3 casos $(3,5,6)$.

A particular localização do mixoma na veia cava inferior, como no presente relato, pode implicar em alguns problemas diagnósticos, bem como cirúrgicos.

A grande contribuição ao diagnóstico dos mixomas foi a utilização da ecocardiografia. Em alguns casos, como no relatado, por não se pensar no diagnóstico ou por falta de boa janela ecocardiográfica, o diagnóstico pode não ser preciso. A utilização da ecocardiografia transesofágica e a tomografia computadorizada de tórax parecem trazer informações adicionais em relação ao ecocardiograma transtorácico.

O tratamento do mixoma é eminentemente cirúrgico. Deve ser instituído tão logo estabelecido o diagnóstico. A manipulação desses tumores deve ser delicada e a boa exposição é obrigatória, no sentido de se identificar não só o sítio de implantação, como também eventuais massas residuais, mesmo em outras câmaras.

A técnica operatória obedece aos conceitos básicos da cirurgia cardíaca. Entretanto, alguns aspectos devem ser levados em consideração no tratamento cirúrgico do mixoma. Antes de qualquer manipulação, é de fundamental importância o pinçamento tanto da aorta quanto do tronco pulmonar, no sentido de se evitar a embolização de fragmentos, visto que os mixomas se constituem de massas gelatinosas e friáveis.

Quanto à localização do mixoma no átrio direito, deve-se realizar a canulação da veias cavas cuidadosamente.

Apesar da característica benigna dos mixomas atriais e do baixo índice de recidiva, o tratamento cirúrgico deve ser o mais agressivo possível. A base de implantação do mixoma deve ser ressecada reconstruindo o septo ou parede atrial com material biológico ou protético (pericárdio bovino ou dacron).

Outro fator de fundamental importância é a investigação de todas as câmaras cardíacas. Além da exposição biatrial, a biventricular também pode ser necessária, no sentido não só de se evitar a recidiva, como também o risco de embolização.

Apesar do caráter benigno dos mixomas intracardíacos, o tratamento radical e o acompanhamento com ecocardiografia periódica é imprescindível.

A localização do mixoma na veia cava inferior, embora muito rara, deve sempre ser lembrada. A sua não identificação pré-operatória, como ocorreu na primeira operação no presente relato, pode levar a planejamento cirúrgico inadequado e incompleta ressecção do tumor. 
Stolf N A G, Benício A, Moreira L F P, Rossi E - Mixoma de átrio direito com origem na veia cava inferior: uma localização rara com implicações diagnósticas e terapêuticas. Rev Bras Cir Cardiovasc 2000; 15(3): 255-8.

\section{RBCCV 44205-510}

Stolf N A G, Benício A, Moreira L F P, Rossi E - Right atrium myxoma originating from the inferior vena cava: an unusual location with therapeutic and diagnostic implications. Rev Bras Cir Cardiovasc 2000; 15(3): 255-8.

ABSTRACT: The myxomas are the most frequent primary cardiac tumors. They are usually located in the left atrium but can be found in other places. This is a case report of a 71 year old patient with diagnosis of a tumor arising from the right atrium, submitted to a surgical resection of the tumor. The operation was realized and the diagnosis confirmed. Resection was successful and the procedure uneventful. Four months postoperatively a standard two-dimensional echocardiogram revealed a residual mass that seemed to arise from the inferior vena cava. He was reoperated and the myxoma originating from the inferior vena cava and extending to the interior of the right atrium was resected. At the basis of the tumor implantation, a portion of the inferior vena cave was resected. The present report shows an unusual location of the myxoma as well as the complications regarding the diagnosis and the approach to surgical treatment.

DESCRIPTORS: Myxoma, surgery. Heart neoplasms, surgery. Vena cava inferior. Myxoma, diagnosis. Heart neoplasms, diagnosis. Vena cava inferior, surgery.

\section{REFERÊNCIAS BIBLIOGRÁFICAS}

1 Bjessmo S \& Ivert T - Cardiac myxoma: 40 year's experience in 63 patients. Ann Thorac Surg 1997; 63: 697-700.

2 Read R C - Cardiac myxoma and surgical history. Ann Thorac Surg 1980; 29: 395-6.

3 Cujec B, Ulmer B, McKaigney et al. - Right atrial myxoma presenting as Budd-Chiari syndrome. Ann Thorac Surg 1987; 44: 658-9.
4 Devig P M, Clark T A, Aaron B L - Cardiac myxoma arising from the inferior vena cava. Chest 1980; 78: 784-6.

5 Bortolotti U, Faggian G, Mazzucco A et al. - Right atrial myxoma originating from the inferior vena cava. Ann Thorac Surg 1990; 49: 1000-2.

6 Selvaraj A, Kumar R, Ravikumar E - Surgical management of the right atrial myxomas: a 15 year experience with review of the literature. J Cardiovasc Surg (Torino) 1999; 40: 101-5. 